\title{
A Study of Optical Sensor based Methods used for Blood Leak Detection During Hemodialysis
}

\author{
Ms. Nandini Rao $\mathrm{G}^{*}$, Ms. Meenakshi $\mathrm{M}^{*}$ and V K Agrawal** \\ "Department of ECE,School of Engineering, DSU \\ ${ }^{* * *}$ Department of CSE,School of Engineering, DSU
}

\begin{abstract}
Blood leakage during hemodialysis can be a serious life-threatening. Venous needle dislodgement can cause a high risk of blood leakage and can be fatal to patients. Intra dialytic blood loss could result from conventional method of blood leak detection. The patient's entrance site ought to be noticeable consistently. It requires a superior, increasingly flexible and effective method of location. A versatile and noninvasive approach of blood leak monitoring is by using photocells. This paper provides the comparative study of various approach used for blood leak detection using photocells or optical sensors.
\end{abstract}

Key words : Venous needle dislodgement (VND), photocells, photointerrupter

\section{INTRODUCTION}

Venous needle dislodgement is an underreported life-threatening complication of hemodialysis[1]. According to the American Nephrology Nurses' Association VND survey reports, VND is a concerning issue for the nephrology nurses/staff and patients. VND can result in severe hemorrhage and can be fatal without rapid response. With blood pump speeds of 350 to $500 \mathrm{~mL} / \mathrm{min}$, VND can result in cardiovascular collapse immediately. Slower leaks of blood around partially dislodged venous needles can also result in significant blood loss. A diagrametic representation of arterivenous fistula is shown in figure 1.Arterial or VND from the access, separation of the venous or blood vessel line associations, femoral or central line dialysis catheter perforation, rupture of a dialysis membrane. Clinical findings include hypotension, loss of consciousness, and cardiac arrest.

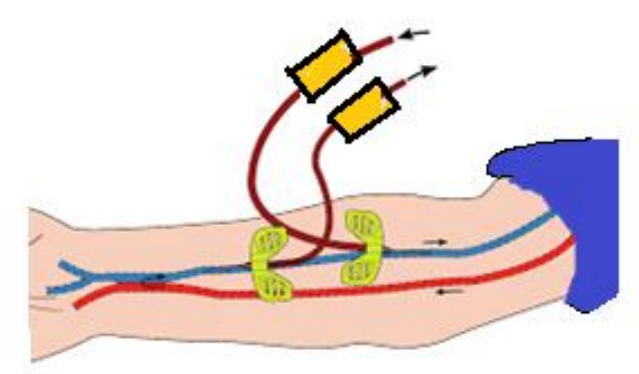

Fig 1:Diagrametic representation of arterivenous fistula

II.BLOOD LEAKAGE IN HEMODIALYSIS

If the blood pump is not stopped, either by activation of the protective system of the dialysis machine or manually, the patient can bleed to death within minutes[1]. The Renal Association Patient Safety Project has identified that renal patients have been exposed to incidents and risks from failure of dialysis equipment or disposables, or from use error. Blood loss on hemodialysis, from venous needle dislodgement as shown in Fig.1 are risks for potential fatal outcomes. Renal patients are susceptible to infection and also are at risk when admitted as acutely ill.

\section{COMPARATIVE STUDY}

The better arrangement discovered is a nonintrusive optical sensor which could be created to distinguish modest quantity of blood in clear liquids on straightforward tubing. These sort of blood loss indicators are structured to such an extent that blood ought not cross the dialysate film. The optical sensor includes an illuminating source with an ideal frequency to give the best affectability in regards to the detection of the red blood cells.
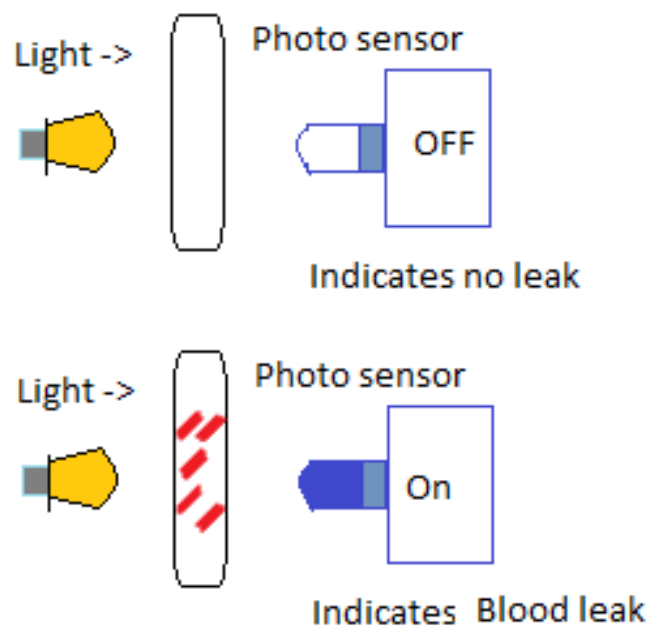

Fig. 2: Using photosensors for blood leak detection.

A simplest approach for blood leak detection is shown in figure 2 . Here photosensors will sense the change in light intensity passing through the tube and detect the blood leakage. But this method requires certain sophisticated conditions to be met such as dark room, no disturbance. Better approach will be using array sensing patch as shown in figure 3 which is combined with a mapping circuit and a wireless module could measure and transmit risk levels [2]. 


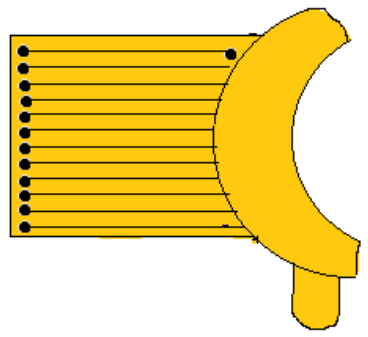

Fig 3:Array sensing patch

Experimental results showed that each point of the sensing array could detect up to $0.1 \mathrm{~mL}$ of leakage of blood and the array sensing patch supports a risk level monitoring system up to $8 \mathrm{~h}$ to alert healthcare personnel of pertinent danger to the patients.

Fog computing with array photocell sensors:

Blood leakage based on fog computing with array photocell sensors and hetero associative memory is used for better monitoring of blood leakage. Photocell sensors are arranged in an array on a flexible substrate as shown in figure 4 to detect blood leakage via the resistance changes with illumination in the visible spectrum of 500-700 $\mathrm{nm}[3]$.

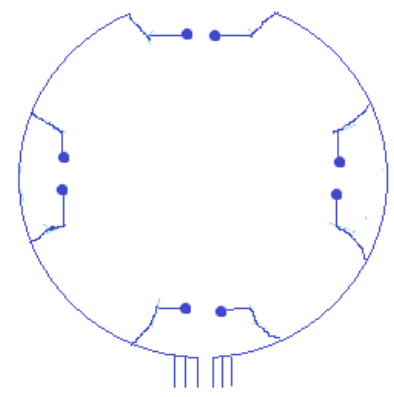

Fig.4: Array of photocell sensor

The hetero associative memory model is implemented with a virtual alarm unit using electricity changes in an embedded system. The warning tool indicates the risk level in both end-sensing units and remote monitor devices via a wireless network and fog/cloud computing.

The absorbent material is placed at the light sensing position of the photo interrupter, which causes the received light intensity to change during blood leakage. Once a blood leakage occurs, the absorbent material absorbs the blood due to the capillary action and triggers the alarm system. A warning light will also be activated, and a leakage occurrence is transmitted to the healthcare stations alarming healthcare workers via the Bluetooth wireless. The healthcare workers can take appropriate action immediately to prevent any risks to the patients during hemodialysis therapy.

A bracelet monitoring device[4] for blood leakage using photo interrupter, bluetooth module, power, and alert components. This needs a very small amount of blood as low as $0.01 \mathrm{ml}$, and $1.6 \mathrm{~s}$ to detect a blood leakage. Furthermore, the lifetime of the battery on this device is longer than the currently available commercial products. It can continuously give out an alert for 18-h long and continuously monitor up to $41 \mathrm{~h}$.

\begin{tabular}{|l|l|l|}
\hline Proposed Method & $\begin{array}{l}\text { Amount of blood } \\
\text { leakage detected }\end{array}$ & Time required \\
\hline Array sensing patch [2] & $0.1 \mathrm{~mL}$ & $90 \pm 5 \mathrm{~ms}$ \\
\hline $\begin{array}{l}\text { Fog computing with } \\
\text { array photocell } \\
\text { sensors[3] }\end{array}$ & $0.1 \mathrm{~mL}$ & $<1 \mathrm{~s}$ \\
\hline $\begin{array}{l}\text { Absorbent material with } \\
\text { photointerrupter[4] }\end{array}$ & $0.01 \mathrm{~mL}$ & $1.6 \mathrm{~s}$ \\
\hline
\end{tabular}

Table 1: Comparative study

A cost effective approach is using an absorbent material which will be placed at the light sensing position of the photointerrupter. It causes the received light intensity to change during blood leakage. Once a blood leakage occurs, the absorbent material absorbs the blood due to the capillary action and triggers the alarm system. A warning light will be activated, and a leakage occurrence will be transmitted to the healthcare stations alarming healthcare workers via the bluetooth. The healthcare workers can take appropriate action immediately to prevent any risks to the patients during hemodialysis therapy.

Conclusion: Using a portable wearable device consisting array of photo sensors to detect blood leak detection would a good solution. But the cost effective approach will be using absorbent material along with photointerrupter. The methods for blood leakage detection can be considered to be to be efficient based on response time and the amount of blood leakage detection within that time as mentioned in Table 1.These methods should be used along with alarm system to indicate the damage. Using IOT for communication, immediate medication will result in efficient blood leak detection system.

\section{REFERENCES}

[1] Ou, Yang-Kun et al. "A Clinical Trial of the Effect of a Blood Leakage Detection Device for Patients during Hemodialysis." International journal of environmental research and public health vol. 16,13 2388. 5 Jul. 2019, doi:10.3390/ijerph16132388

[2] Du, Yi-Chun et al. "Novel Wearable Device for Blood Leakage Detection during Hemodialysis Using an Array Sensing Patch.” Sensors (Basel, Switzerland) vol. 16,6 849. 9 Jun. 2016, doi:10.3390/s16060849

[3] Wu, Jian-Xing et al. "Blood leakage detection during dialysis therapy based on fog computing with array photocell sensors and heteroassociative memory model." Healthcare technology letters vol. 5,1 38-44. 26 Jan. 2018, doi:10.1049/ht1.2017.0091

[4] Chuang, Ho-Chiao \& Shih, Chen-Yu \& Chou, Chin-Hui \& Huang, Jung-Tang \& Wu, Chih-Jen. (2015). The Development of a Blood Leakage Monitoring System for the Applications in Hemodialysis Therapy. Sensors Journal, IEEE. 15. 1515-1522. 10.1109/JSEN.2014.2364302.

[5] Lin, Chia-Hung et al. "Detection of venous needle dislodgement during haemodialysis using fractional order shape index ratio and fuzzy colour relation analysis." Healthcare technology letters vol. 2,6 149-55. 19 Nov. 2015, doi:10.1049/ht1.2015.0022

[6] Ho-Chiao Chuang, Chen-Yu Shih, Chin-Hui Chou, Jung-Tang Huang, and Chih-Jen Wu "The Development of a Blood Leakage Monitoring System for the Applications in Hemodialysis Therapy", IEEE SENSORS JOURNAL, VOL. 15, NO. 3, MARCH 2015

[7] Victor F. Seabra, Bertrand L. Jaber, in Comprehensive Clinical Nephrology (Fourth Edition), 2010,vol 6 no 1 January 2014 journal of renal nursing 